Cuadernos de Lingüística Hispánica n. ${ }^{\circ} 31$

ISSN 012 I-053X • ISSN en línea 2346- 1829

Enero - Junio 2018, pp. 37-57

\title{
Multilingüismo y exogamia en la etnia tucana del Vaupés*
}

\author{
RAMIRO NEIL ROJAS APRÁEZ ${ }^{* *}$ \\ MARISOL AVENDAÑO CEPEDA**
}

Recepción: 22 de junio de 2017

Aprobación: 10 de agosto de 2017

Forma de citar este artículo: Rojas, R.N. \& Avendaño, M. (2018). Multilingüismo y exogamia en la etnia tucana del Vaupés. Cuadernos de Lingüística Hispánica, (31), 37-57. doi: https://doi.org/10.19053/0121053X.n31.2018.7742

* Artículo de investigación que presenta los resultados del proyecto de investigación titulado "Exogamia y multilingüismo en la etnia tucana del departamento del Vaupés", para optar al título de magíster en Lingüística.

** Magíster en Lingüística, UPTC. Licenciado en Ciencias Sociales, Universidad Pedagógica Nacional (Bogotá). Diplomado en Traducción y Alfabetización, Universidad Ricardo Palma (Lima, Perú). Actualmente, docente en secundaria, Colegio El Duque de Rivas, Bogotá. Correo electrónico: apraez07@gmail.com.

** Magíster en Lingüística, UPTC. Licenciada en Español e Inglés, Universidad Pedagógica Nacional, (Bogotá). Especialización en Administración Educativa, Universidad de La Sabana, Chía. Diplomado en Alfabetización y Traducción, Universidad Ricardo Palma, Lima-Perú. Actualmente, rectora y docente en el Colegio El Duque de Rivas, Bogotá. Correo electrónico: rosavendanio@gmail.com. 


\section{Resumen}

El multilingüismo es un fenómeno sobresaliente en la etnia tucana. El objetivo de la investigación fue describir y analizar el estado actual de este rasgo, a fin de establecer los aspectos lingüísticos y antropológicos que intervienen en este fenómeno. Esta investigación se enmarcó dentro de los límites de la sociolingüística y la antropología, con un enfoque cualitativo y un método etnográfico, en donde el trabajo in situ fue fundamental, y la observación, el diálogo y la entrevista fueron las herramientas puntuales. Al analizar los resultados se evidenció que la etnia tucana continúa respetando la regla primigenia de la exogamia, ya que no fueron reportados casos de familias establecidas bajo relaciones endogámicas, y es la exogamia la que aún mantiene vivo el multilingüismo en medio de los tucanos. Se estableció que los miembros de esta etnia poseen la habilidad de comunicarse sin dificultad con personas de otras etnias, entre ellas: sirianos, desanos y cubeos, y se observó al recorrer las comunidades tucanas ubicadas en Brasil que los tucanos colombianos podían entender también el portugués.

Palabras clave: sociolingüística, lenguas en contacto, multilingüismo, etnia tucana, antropología, exogamia.

\section{Multilingualism and Exogamy in the Tucano Ethnic Group from Vaupes, Colombia}

\section{Abstract}

Multilingualism is a prominent phenomenon in the Tucano ethnic group. The main purpose of this research is to describe and analyze the current state of the affairs regarding this specific feature of Tucano culture, in order to identify linguistic and anthropological aspects that play a role in this phenomenon. This research is set within the frame of Sociolinguistics and Anthropology, employing a qualitative approach, and an ethnographic method based on field work. The main research tools were: participant observation, conversation, and interviews. Given the fact that there is not a single reported case of a family established by endogamic relationship, it can be proved that the Tucano ethnic group still respects the primal rule of exogamy, thus keeping alive the multilingualism among the Tucanos. It was also evidenced that the members of this ethnic group have the ability to communicate effortlessly with people from other cultures, such as the Sirianos, the Desanos and the Cubeos. Finally, an expedition through the Tucano communities located in Brazil showed that Colombian Tucanos were also able to understand Portuguese.

Keywords: sociolinguistics, language contact, multilingualism, Tucano ethnic group, Anthropology, exogamy. 


\section{Multilinguisme et exogamie dans l'ethnie tucana du Vaupés}

\section{Résumé}

Le multilinguisme est un phénomène remarquable dans l'ethnie tucana. L'objectif de la recherche a été celui de décrire et analyser l'état actuel de ce trait, afin d'établir les aspects linguistiques et anthropologiques qui interviennent dans ce phénomène. Cette recherche s'est inscrite dans les limites de la sociolinguistique et l'anthropologie, avec une approche qualitative et une méthode ethnographique, où le travail in situ a été fondamental, et l'observation, le dialogue et l'entretien ont été des outils ponctuels. Lors de l'analyse des résultats, il a été mis en évidence que l'ethnie tucana continue de respecter la règle de l'exogamie, puisqu'on n'a pas rapporté des cas de familles établies sous des rapports endogamiques, et c'est l'exogamie celle qui maintient le multilinguisme encore vivant au milieu des tucanos. On a établi que les membres de cette ethnie possèdent l'habileté de communiquer sans difficulté avec des personnes d'autres ethnies, parmi lesquelles: sirianos, desanos et cubeos, et on a observé, en parcourant les communautés tucanas situées au Brésil, que les tucanos colombiens pouvaient aussi comprendre le portugais. .

Mots clés: sociolinguistique, langues en contact, multilinguisme, ethnie tucana, anthropologie, exogamie. .

\section{Multilingualismo e exogamia na etnia tucana do Vaupés}

\section{Resumo}

O multilingualismo é um fenômeno notável no grupo étnico Tucano. 0 objetivo da pesquisa foi descrever e analisar o estado atual desta característica, a fim de estabelecer os aspectos linguísticos e antropológicos que intervêm neste fenômeno. Esta pesquisa foi enquadrada dentro dos limites da sociolinguística e da antropologia, com abordagem qualitativa e método etnográfico, onde 0 trabalho in situ era fundamental, e observação, diálogo e entrevista eram as ferramentas específicas. Ao analisar os resultados, ficou evidente que 0 grupo étnico Tucano continua a respeitar a regra original da exogamia, uma vez que não houve casos relatados de famílias estabelecidas sob relações de endogamia, e é a exogamia que mantém o multilingualismo vivo no meio dos Tucanos. Foi estabelecido que os membros deste grupo étnico possuem a capacidade de se comunicar sem dificuldade com pessoas de outros grupos étnicos, incluindo: sírianos, desanos e cubeos, e foi observado ao visitar as comunidades Tucanas localizadas no Brasil que os tucanos colombianos também podem entender o português.

Palavras-chave: sociolinguística, línguas em contato, multilingualismo, etnia tucana, antropologia, exogamia. 


\section{Introducción}

El estudio de la exogamia y el multilingüismo en la comunidad tucana del Vaupés se abordó desde la perspectiva de la sociolingüística y la antropología, con el fin de establecer qué elementos de tipo sociolingüístico y antropológico intervienen en la conservación del multilingüismo en esta comunidad. Desde la sociolingüística, la investigación se focalizó en el área del contacto de lenguas, y desde la antropología con base en el estudio de las relaciones de parentesco, se centró en lo pertinente a la exogamia.

\section{Caracterización de la etnia tucana}

Los tucanos se encuentran asentados en pequeñas comunidades ubicadas en el departamento del Vaupés, en un territorio comprendido entre la llanura de la Orinoquía colombiana y la selva húmeda de la Amazonía. El área geográfica específica en donde se realizó la investigación corresponde al suroriente colombiano, en la frontera con Brasil; allí se visitaron tres comunidades tucanas (Acaricuara, La Floresta y Tamacuarî), ubicadas en las orillas del río Paca y del río Papurí, las cuales se seleccionaron por sus características, entre ellas su accesibilidad.

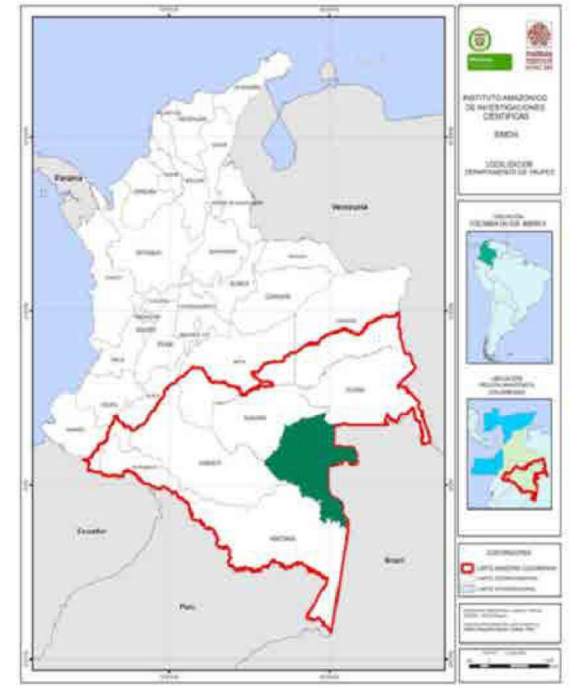

Figura 1. Mapa de ubicación del departamento del Vaupés.

Fuente: Instituto Amazónico de Investigaciones Científicas.

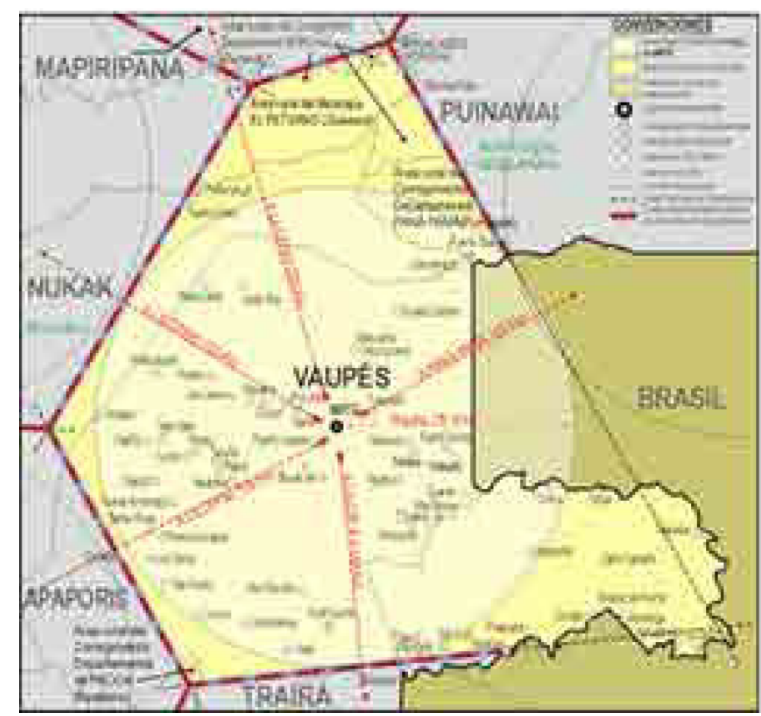

Figura 2. Mapa de comunidades del departamento del Vaupés.

Fuente: Salamanca Parra (2010).

Los tucanos viven en pequeñas comunidades, cuyos territorios están amparados, según lo establecido en el artículo 329 de la Constitución Política nacional, bajo la figura del resguardo; están liderados por un capitán, máxima autoridad en la comunidad, quien obtiene este cargo a través de un proceso de elección popular para un periodo de un año. 
Los tucanos son pacíficos por naturaleza y tienen buenas relaciones con los grupos vecinos, entre ellos los desanos, los sirianos y los cubeos; sus familias son nucleares, con una figura paterna, que asume el liderazgo en la toma de decisiones, y una figura materna, que desempeña roles como la preparación de los alimentos, el cuidado de los hijos y el trabajo en la chagra (huerta o parcela familiar en donde se cultiva).

La actividad económica de los tucanos se basa en la agricultura, la cual practican en la chagra, donde siembran yuca brava, yuca dulce, maíz, pupuña (fruta que en algunas zonas de Colombia se conoce como chontaduro), plátano, caña de azúcar, piña, papaya y varias clases de tubérculos. Los productos obtenidos son para el consumo familiar, ya que la producción no es suficiente para comercializarlos. El programa Familias Guardabosques les proporcionó durante algún tiempo los insumos necesarios para desarrollar proyectos como el cultivo de ají, piña y la cría de algunos animales. Además practican la caza de dantas, monos, cerdos salvajes, roedores, monos, ardillas y aves, para lo cual usan la escopeta, también pescan utilizando instrumentos como el arpón, el anzuelo y la red.

La base de su alimentación es la yuca brava, con la que, una vez extraído el veneno que contiene, elaboran enormes tortas denominadas casabe (pan tradicional preparado con la harina de la yuca brava). Su preparación es un proceso culinario dispendioso, el cual debe ser desarrollado paso a paso y con mucho cuidado.

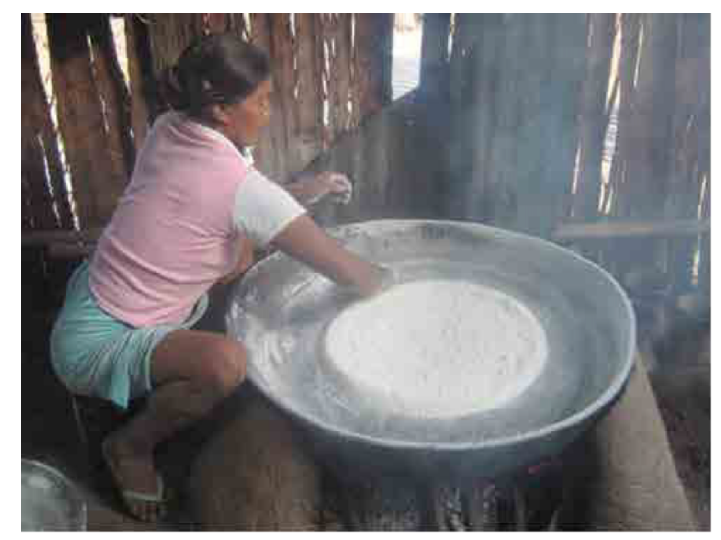

Figura 3. Fotografía de señora preparando la torta de casabe.

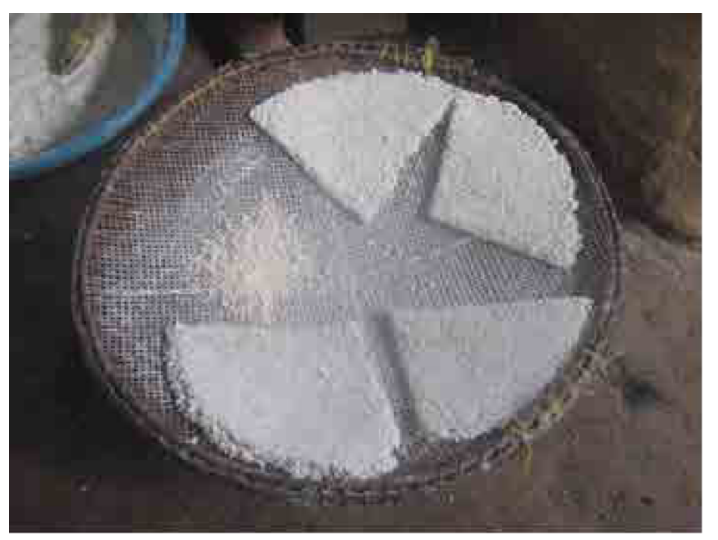

Figura 4. Casabe porcionado.

Los tucanos fabrican diferentes elementos para las tareas diarias y también elaboran artesanías como canastos y pequeñas sillas talladas a partir de un tronco, el cual van moldeando hasta darle la forma deseada. Estas sillas pueden medir entre veinte y treinta centímetros de altura, y se constituyen en la artesanía representativa de esta etnia. Debido al intercambio familiar y cultural con las etnias cercanas es común aprender y elaborar 
otro tipo de artesanías, las cuales son puestas a la venta en las cabeceras municipales del departamento 0 en ferias artesanales.

En relación con el aspecto educativo y con base en la información recibida en las reuniones con docentes y directivos docentes, fue posible determinar que en las comunidades tucanas existe cobertura educativa en los ciclos de básica primaria, básica secundaria y media vocacional. En ellos se procura impartir una educación bilingüe: empleando la lengua materna durante los primeros años y luego el español en forma gradual; aunque surge un problema cuando, en no pocas ocasiones, los maestros indígenas son enviados a escuelas en donde se habla una lengua diferente a la suya y esto les obliga a enseñar a los niños pequeños en español. En las comunidades más grandes, como Acaricuara y Monfort, hay actualmente instituciones que ofrecen los tres ciclos y que cumplen la función de internados, hasta allí se desplazan los niños que han culminado la básica primaria en sus comunidades. Los materiales escritos en lengua materna son muy escasos, casi exiguos, lo cual dificulta aún más el uso de la lengua en el contexto escolar.

Los siguientes son algunos rasgos sobresalientes de la lengua tucana: pertenece a la familia lingüística tucano oriental; posee seis vocales "orales": a,e,i,o,u,, y seis vocales nasales: ã,ẽ,Ĩ,õ,ũ,ũ; cuenta con diecinueve consonantes que se representan con los siguientes grafemas: b,c,cj,d,g,j,m,n,ñ,p ,pj,q,r.s,t,tj,w,y,' (oclusiva glotal); emplea dos tonos, uno alto y uno bajo, ortográficamente solo se marca el tono alto. Otro elemento llamativo es el uso de un tiempo pasado "reportativo" y uno evidencial, este último se emplea cuando quien "cuenta" lo sucedido realmente lo vio o participó en él. En palabras de los propios tucanos, su lengua es una "lengua de nariz", es decir, con rasgos nasales muy marcados (West \& Welch, 2004).

Los tucanos son animistas, por eso dentro de sus creencias y de su cultura espiritual la historia de la "Gran boa canoa" ocupa un lugar especial, pues a través de ella explican el origen de la etnia tucana y de los otros grupos habitantes de la tierra del Vaupés.

\section{Referentes teóricos}

Los estudios de lenguas en contacto se empiezan a desarrollar con rigurosidad a partir de los años cincuenta, con el trabajo realizado por Uriel Wenreich: Lenguages in Contact (1953); en el siglo XIX ya hay una aproximación al estudio, cuando William Dwight (1881) debatió de forma explícita la función de los préstamos en el campo lingüístico, y de igual manera Hugo Schuchardt (1881) documentó en varias publicaciones situaciones complejas de contacto. Schuchardt es el precursor del estudio de las lenguas criollas. 
Se define el contacto de lenguas como el lugar donde confluyen dos o más lenguas. El lingüista mexicano Sala (1988) dice que el contacto de lenguas puede darse de manera directa, cuando dos o más lenguas son usadas en una comunidad, o de manera indirecta, cuando no se usa la lengua en una determinada comunidad, pero sí se tiene contacto con ella a través de la educación, de los medios de comunicación o de documentos.

Para Iriarte Esguerra (citado en Ramírez, 2009), el contacto de lenguas se centra en la interconexión entre diferentes lenguas del mundo y en sus desarrollos históricos, con el fin de analizar temas relacionados con su evolución y cómo a partir de una lengua primigenia se originan nuevas lenguas. Iriarte también señala la forma en la que el desarrollo de las lenguas influye en el contacto cultural entre hablantes de distintas procedencias linguísticas (Ramírez, 2009). Por su parte, Weinreich (1974) indica que el contacto de lenguas se presenta en el instante en que dos o más lenguas son utilizadas alternativamente por las mismas personas.

Ahora bien, los estudios del contacto de lenguas se orientan en dos líneas de investigación; una primera línea, que se centra principalmente en el origen de los sistemas lingüísticos, que se encuentran en una lengua, con el fin de establecer similitudes presentes en lenguas de diferente naturaleza; y una segunda línea de investigación, que apunta al estudio de contextos sociales, centrándose en las relaciones de grupo y cómo las lenguas se acomodan o divergen bajo ciertas circunstancias de orden político, económico, social y cultural.

En este orden de ideas, el contacto de lenguas presenta el bilingüismo como una de sus manifestaciones, "el contacto de lenguas conduce inevitablemente al bilingüismo" (Appel \& Muysken, 1996, p. 10). La definición clásica de bilingüismo establece que bilingüe es aquella persona que tiene dominio pleno de dos lenguas. Frente a esta definición existen varias posiciones que estructuran sus conceptos de acuerdo con un criterio psicológico o sociológico. Según Appel y Muysken (1996), el criterio psicológico hace alusión a la competencia lingüística, y el sociológico se refiere al uso alternativo de dos lenguas (citados por Weinreich, 1974, p. 5). El primer criterio se centra en la capacidad del individuo para aprender las lenguas, y el segundo, en el uso que hace de las lenguas en su comunidad. Por su parte, Silva-Corvalán (1989) afirma que el bilingüismo se presenta cuando los miembros de una comunidad utilizan dos o más lenguas.

A lo largo de la historia y en momentos particulares han existido y existen personas, grupos y culturas que han trascendido el uso de dos sistemas lingüísticos, es decir, no solo han aprendido dos lenguas, sino que hablan tres o más lenguas. En estos casos se habla de personas 0 comunidades trilingües 0 , utilizando un concepto más amplio, plurilingües 0 
multilingües (Siguan, 2011). En cuanto al multilingüismo, este se da cuando un hablante hace uso de tres o más lenguas (López, 2000). Anokhina, a su vez, describe el hecho de que una persona 0 una comunidad sea multilingüe, es decir, que sea capaz de expresarse en varias lenguas (Anokhina, 2013).

De acuerdo con el estudio de la exogamia, esta se circunscribe al campo conceptual de la antropología de parentesco o estudio de parentesco que, a su vez, se enmarca dentro de la antropología social. Se define la exogamia como una regla que rige para la elección del cónyuge y que prohíbe las relaciones matrimoniales entre los miembros de un mismo grupo. Los primeros trabajos de endogamia y exogamia fueron realizados en la primera mitad del siglo XIX, cuando Sir George Grey realizó sus primeras observaciones con tribus australianas.

Para Grey (1841), la exogamia y la endogamia son formas de reglamentación en los sistemas de parentesco, pero es John Ferguson McLennan, con su trabajo Primitive marriage publicado en 1865 (citado por Martínez, 2008), quien empieza a desarrollar y ampliar los conceptos de endogamia y exogamia en los sistemas de parentesco. Lewis $\mathrm{H}$ Morgan en el año 1879, con su trabajo Systems of Consaguinity and Afinity of Human Family (Martínez, 2008), establece el papel central de la exogamia en la evolución de las sociedades humanas. Y por su parte, Claude Levi-Strauss en su texto Estructuras elementales del parentesco, presenta un panorama del sistema de parentesco a partir de cuatro ejes temáticos: la naturaleza y la cultura, el incesto, las reglas y la endogamia y la exogamia (Levi Strauss, 1985).

\section{Metodología}

La población objeto de estudio fueron los tucanos. El área geográfica de investigación fue el departamento del Vaupés, ubicado al suroriente de Colombia, en la frontera con Brasil. Como ya se describió, los tucanos se organizan en pequeñas comunidades, bajo la figura del resguardo, dirigidos por un capitán, quien es escogido a través de un sistema de elección popular, para un periodo de un año. Se trabajó con tres comunidades tucanas: Acaricuara, La Floresta y Tamacuari, ubicadas en las orillas del río Paca y del río Papuri.

Se dialogó inicialmente con algunos miembros de la comunidad, con el fin de explicar nuestro propósito y generar un ambiente de confianza; posteriormente y con la autorización de los capitanes, se aplicaron las encuestas. Fueron encuestadas cuarenta y seis personas, veinte de la comunidad de Acaricuara, quince de la Floresta y once de Tamacuari, los que respondieron eran jefes cabeza de familia, en su mayoría hombres, quienes hablan con propiedad del tema propuesto, debido al papel de autoridad que tienen dentro 
de su núcleo familiar y de la comunidad en general. Es importante señalar que antes de la aplicación de la encuesta se hizo una serie de contactos y visitas que garantizaban la elaboración de un plan de trabajo apropiado.

Las herramientas utilizadas en la recolección de datos fueron: la observación, la entrevista informal y la participación en eventos y actividades comunitarias. En la entrevista se diseñaron veinte preguntas, que se relacionan con dos categorías: lengua y familia. De estas dos categorías surgieron ocho subcategorías, que son: etnias con las cuales establecen parentesco los tucanos, lenguas que entienden, lenguas que hablan, espacio de adquisición de la lengua más empleada, espacios de uso de las diferentes lenguas, uso del tucano frente a extraños, lenguas aprendidas y habladas por los hijos y lengua que se emplea en la escuela.

\section{RELACIÓN EXOGAMIA Y MULTILINGÜISMO EN LA ETNIA TUCANA DEL DEPARTAMENTO DEL VAUPÉS}

Encuesta número: Comunidad: Fecha:

1.Nombre:

2.Fecha de nacimiento: 3. Edad:

4.Lugar de nacimiento:

5.Lengua (s) que habla:

6.Lengua (s) que entiende:

7.Etnia a la que pertenece: 7.1 Estado civil: casado soltero

8.Nombre del cónyuge:

9.Etnia del cónyuge:

10.Lengua (s) que habla:

11.Lengua (s) que entiende:

12.Número de hijos: 
Datos de los hijos:

\begin{tabular}{|c|c|c|c|c|c|c|c|}
\hline $\mathrm{N}^{0}$ & Nombre & $\begin{array}{c}\text { Edad } \\
\text { (años) }\end{array}$ & $\begin{array}{c}\text { Lugar de } \\
\text { nacimiento }\end{array}$ & Lenguas & $\begin{array}{l}\text { ¿Dónde la } \\
\text { aprendió? }\end{array}$ & $\begin{array}{l}\text { Estudia } \\
\text { Sí-No }\end{array}$ & $\begin{array}{l}\text { ¿En cuál } \\
\text { lengua? }\end{array}$ \\
\hline 1 & & & & & & & \\
\hline 2 & & & & & & & \\
\hline 3 & & & & & & & \\
\hline 4 & & & & & & & \\
\hline 5 & & & & & & & \\
\hline 6 & & & & & & & \\
\hline 7 & & & & & & & \\
\hline 8 & & & & & & & \\
\hline 9 & & & & & & & \\
\hline 10 & & & & & & & \\
\hline
\end{tabular}

Sobre el aprendizaje y uso de la lengua:

13.¿Cuál lengua habló inicialmente (primero)?

14.¿Dónde aprendió a hablar la lengua tucana?

15.¿En qué lengua escribe?

16.¿Qué lengua utiliza con más frecuencia en:
a.La casa
b.La chagra
c.En el trabajo comunitario
d.En las reuniones de la comunidad
e.En Mitú

f. En la iglesia

17. ¿Habla en tucano frente a personas extrañas? Sí No

18. ¿Por qué?

19. ¿Qué ventajas tiene para usted el hablar o entender varias lenguas?

20. Por favor describa su comunidad 
De acuerdo con Gaskel (2002), la entrevista cualitativa es un intercambio de ideas, significados y sentimientos sobre el mundo y los eventos, cuyo principal medio son las palabras. Esta herramienta es la más eficaz y recurrente en el trabajo en las comunidades indígenas. Para ellas, el diálogo es la forma apropiada de intercambiar saberes, de compartir y conocer otras formas de vida. Debido al ritmo dentro de cada grupo, las tardes, alrededor de una torta de casabe, y las reuniones comunitarias, son los espacios más propicios para las entrevistas informales.

\section{Resultados y análisis}

Con la primera subcategoría fue posible determinar los vínculos que establecen los tucanos con las diferentes etnias: con los sirianos, los desanos, los barasanos y los tuyucas, entre otros; situación fundamental para considerar la continuidad del multilingüismo. Al analizar los resultados obtenidos en la primera subcategoría es posible precisar que dentro de las tres comunidades tucanas observadas, visitadas y encuestadas, la práctica de la exogamia de tipo patrilineal continúa vigente en la actualidad, lo cual reafirma lo dicho por la investigadora Jackson (1983). Todos los encuestados establecieron parentesco con miembros de otras etnias, siendo la etnia siriana la que predomina en el momento de establecer vínculos matrimoniales, luego, en orden descendente, continúan las etnias desana, tuyuca, barasana y cubea. Si se relacionan los resultados con la ubicación geográfica de las etnias mencionadas es posible determinar que la cercanía puede ser la razón primordial para establecer el mayor número de vínculos familiares.

\section{CONSOLDADO}
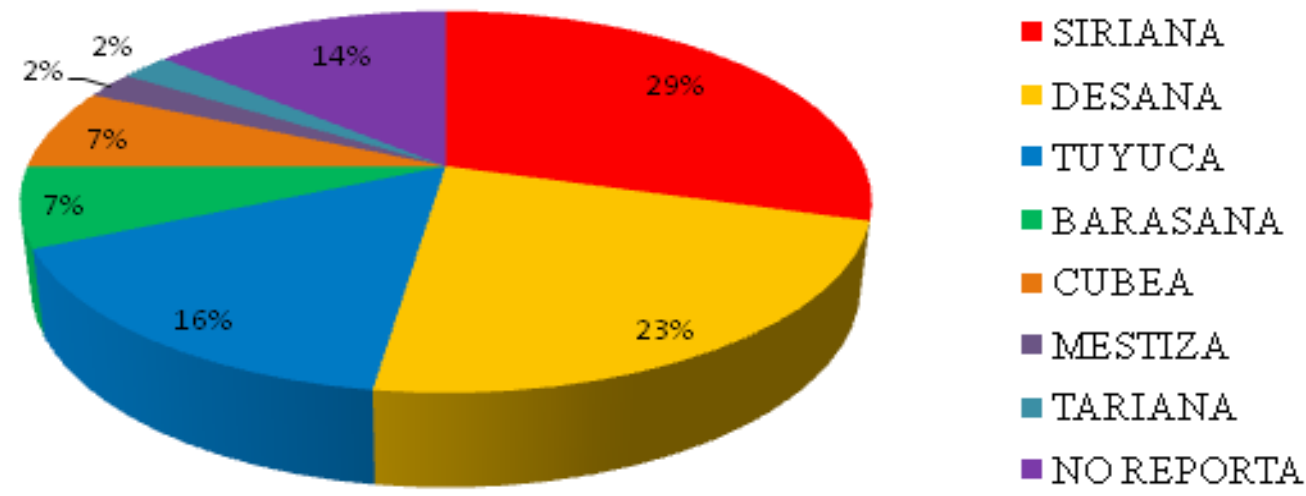

Figura 5. Etnias con las cuales establecen parentesco los tucanos.

Se propuso la subcategoría lenguas que entienden, para establecer una diferencia con las lenguas que hablan, ya que muchos hablantes tucanos entienden varias lenguas, pero cuando hablan no las emplean todas, prefieren algunas. Los resultados señalan que 
hay dos lenguas que todos los miembros encuestados de estas comunidades comprenden: el tucano y el español; lo que permite establecer una buena convivencia entre la lengua materna (el tucano) y la lengua nacional (el español). Además de entender el tucano y el español, algunos miembros de las tres comunidades tucanas encuestadas pueden entender el siriano, el desano, el tuyuca, el barasano, el carapana, el yuruti, el cubeo, el guanano, el piratapuyo, el bará y el curripaco; y aunque no lo registraron en las encuestas, fue posible observar que al recorrer las comunidades tucanas ubicadas en el Brasil, los tucanos que guiaron los viajes podían entender también el portugués.

Los resultados obtenidos y ubicados dentro de la subcategoría dos están directamente relacionados con la categoría uno, ya que al continuar con la práctica de la exogamia es propio de los hijos hablar la lengua del padre y, a su vez, estar en el ambiente propicio para aprender también la lengua de la madre, como afirma Landaburu (2004):

El principio básico es que no me puedo casar con una mujer (o un hombre) de lengua A cuyo padre sea de la misma lengua que mi padre. Dos hombres "definidos" por la misma lengua son vistos como consanguíneos. La hija de uno de ellos no puede ser dada al hijo del otro. Sería incestuoso. Además, como mi madre no era de lengua $\mathrm{A}$, ella me enseñó su lengua, por ejemplo $\mathrm{B}$. Los hermanos de mi padre, con quienes vivo, han podido casar mujeres de lengua $\mathrm{B}$ o $\mathrm{C}$ o $\mathrm{D}$, lenguas que he oído de pequeño y que normalmente domino. Soy por lo tanto bilingüe, trilingüe 0 cuatrilingüe desde niño. Eso me permite casarme sin dificultades de comunicación con una mujer que sea de lengua B, C o D. (p. 10).
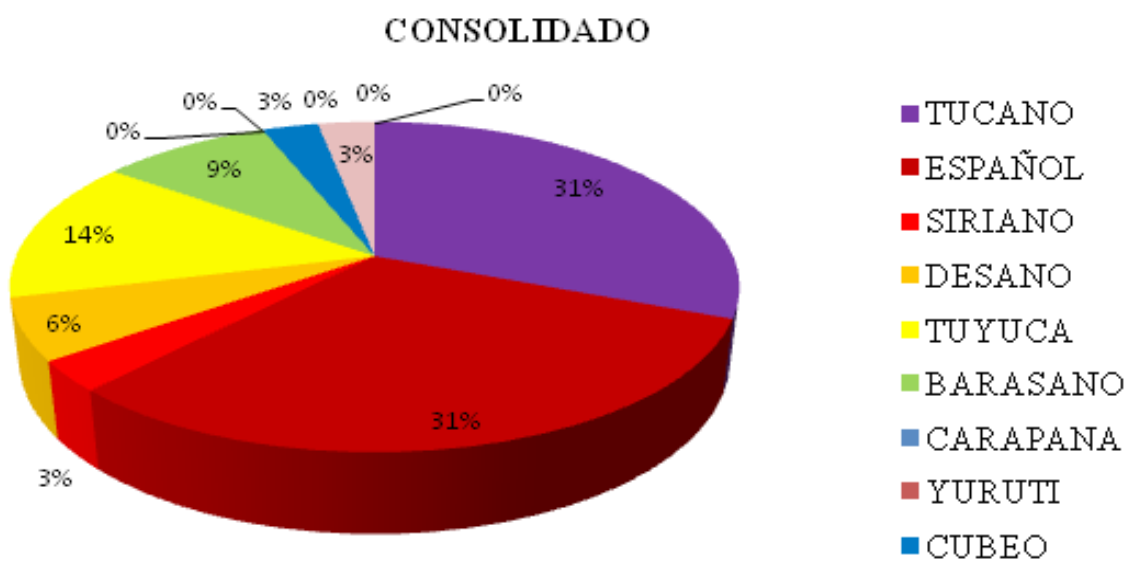

Figura 6. Lenguas que entienden.

Los resultados indicaron que las lenguas que más hablan, además del tucano, son, en su orden: español, siriano, tuyuca, piratapuyo, yurutí y curripaco. Si bien manifiestan entender muchas más lenguas, en la práctica, al comunicarse prefieren utilizar el tucano y el español; lo cual confirma lo manifestado y escuchado en la región durante los viajes, 
que el tucano es considerado por los habitantes de la región como una lengua franca 0 comercial.

\section{CONSOLIDADO}

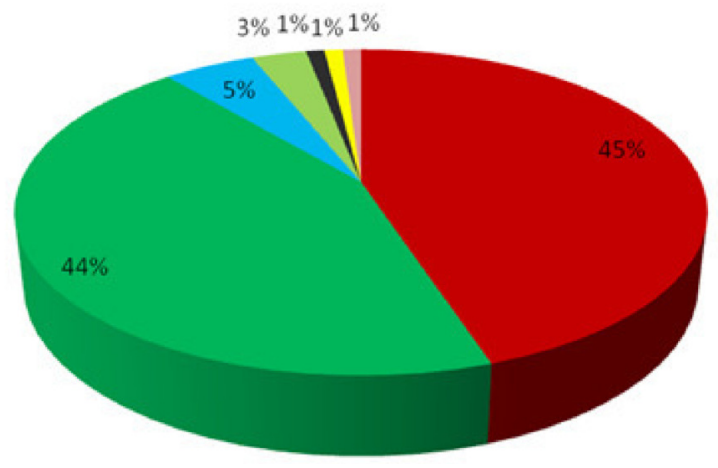

- TUCANO

- ESPAÑOL

$\because$ SIRIANO

$\because$ TUYUCA

- PIRATAPUYO

YURUTI

= CURRIPACO

Figura 7. Lenguas que hablan los tucanos.

En los espacios de adquisición de la lengua más empleada se buscó establecer en qué contextos aprendieron la lengua que más usan, y al respecto se pudo determinar que la casa es el lugar donde aprendieron la lengua, y que es la lengua paterna la que se asimila en el hogar. Los padres son las personas encargadas socialmente de desarrollar de manera natural esta importante tarea, la de transmitir la lengua, y con ella la cultura, a las nuevas generaciones. De esta forma se asegura la pervivencia de la lengua tucana en la región. Esa transmisión se da de forma oral, y es en el diario vivir y transcurrir que los hijos reciben el conocimiento no solo de la lengua sino de la cultura; y a la casa le siguen en su orden, la comunidad y la escuela.

TABLA GLOBAL

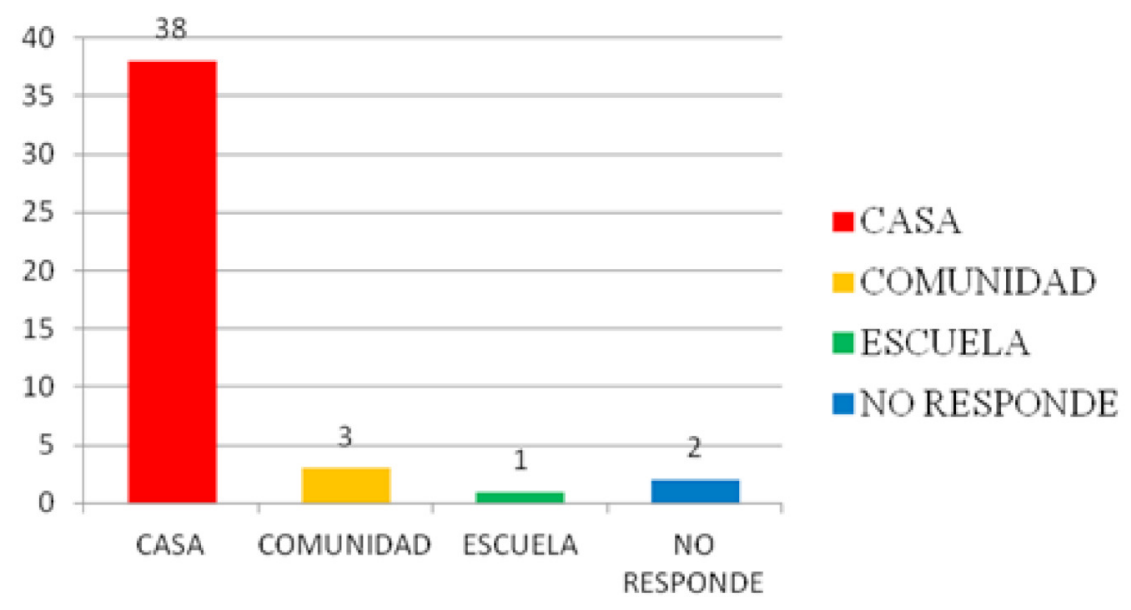

Figura 8. Espacio de adquisición de la lengua más empleada. 
En la siguiente subcategoría se buscaba verificar los espacios de uso de las diferentes lenguas; de acuerdo con la escala de Fishman (1995), de ello depende, en gran medida, la posibilidad del mantenimiento de una lengua, ya que según el lugar de uso, la ocasión de vigencia puede ser mayor o menor. Los espacios propuestos a los encuestados se determinaron previamente observando las costumbres de los miembros de la comunidad. Estos lugares fueron: la casa, la chagra, el espacio de trabajo comunitario (que varía de acuerdo con la ocasión y la necesidad), la ciudad de Mitú y la iglesia. Al analizar los resultados estos se pueden agrupar en dos bloques: en el primero se emplea la lengua tucana, y los lugares de uso son: la casa, la chagra, el espacio de trabajo comunitario y las reuniones de la comunidad. En el segundo se utiliza el español y los lugares de uso son: la ciudad de Mitú y la iglesia.

\section{ACUMULADO}

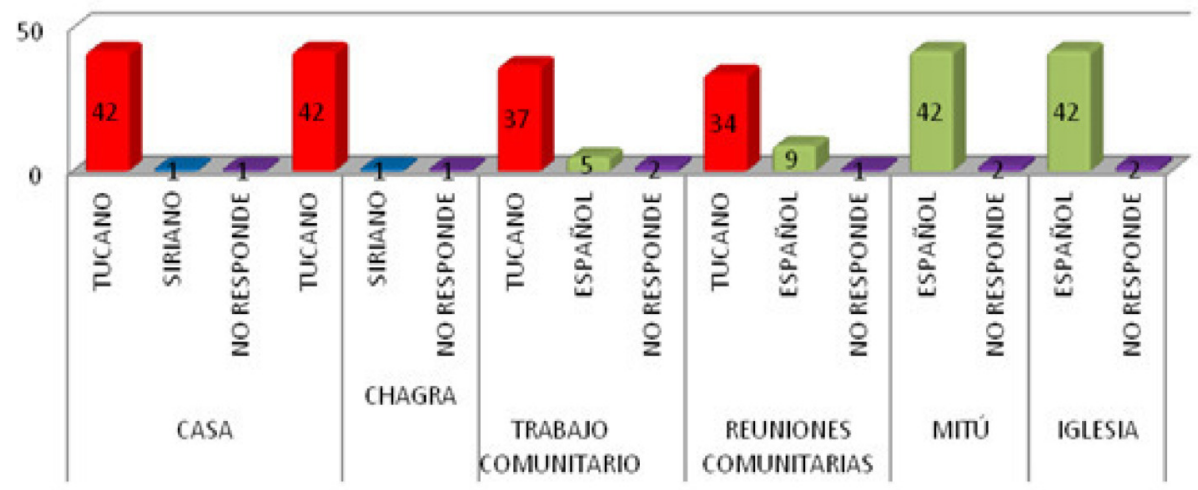

Figura 9. Espacio de uso de las diferentes lenguas.

La pregunta sobre el uso de la lengua tucana frente a extraños buscaba evidenciar la actitud y el grado de confianza de los encuestados al emplear la lengua materna ante personas que no pertenecen a la comunidad y las razones que consideran pertinentes para usarla 0 abstenerse de hacerlo. Los resultados obtenidos mostraron que en comunidades como Acaricuara y la Floresta, un $90 \%$ y un $82 \%$ respectivamente usaban la lengua frente a personas que no hablaban tucano. Las razones que esgrimieron fueron: porque otros necesitan aprender nuestra lengua, porque ellos la entienden, para familiarizarse con otras personas, porque ellos quieren aprender y porque la lengua tucana es natural.

Los resultados obtenidos en la comunidad de Tamacuari fueron opuestos a los de las dos comunidades anteriores, allí solo el $18 \%$ de los encuestados hablaban tucano frente a personas extrañas. Las razones que dieron para no hablar tucano frente a personas 
que no son de la comunidad son: no entienden la lengua, no nos entendemos y para no hacer quedar mal a las personas que no los entienden.

\begin{tabular}{|c|c|c|c|}
\hline \multicolumn{2}{|c|}{ ACUMULADO } & \multicolumn{2}{|c|}{ ACUMULADO } \\
\hline Sí & 28 & SÍ & $64 \%$ \\
\hline NO & 16 & NO & $36 \%$ \\
\hline
\end{tabular}

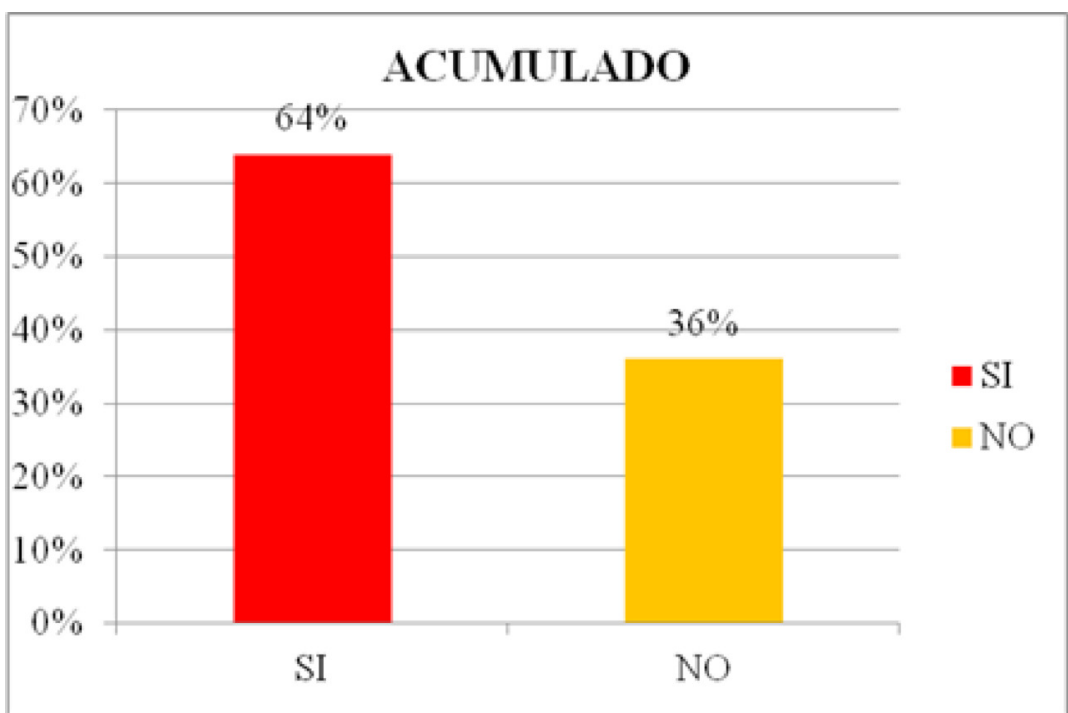

Figura 10. Uso de la lengua materna: tucano, frente a extraños.

De las lenguas que hablan los hijos se buscó inferir la continuidad del multilingüismo en las nuevas generaciones. Para una mejor comprensión de los resultados en esta categoría, se presentan los resultados por comunidad. Al analizar los resultados se encontró que en Acaricuara predomina entre los hijos el conocimiento y el uso de la lengua tucana, siguiendo el español y finalmente el siriano.

\begin{tabular}{|c|c|c|c|c|c|c|c|c|c|c|}
\hline \multicolumn{10}{|c|}{ Acaricuara } \\
\hline $\begin{array}{c}\text { Familia } \\
1\end{array}$ & \multicolumn{2}{|c|}{$\begin{array}{c}\text { Familia } \\
2\end{array}$} & $\begin{array}{c}\text { Familia } \\
3\end{array}$ & \multicolumn{2}{|c|}{$\begin{array}{c}\text { Familia } \\
4\end{array}$} & \multicolumn{3}{|c|}{$\begin{array}{c}\text { Familia } \\
5\end{array}$} & $\begin{array}{c}\text { Familia } \\
6\end{array}$ & $\begin{array}{c}\text { Familia } \\
7\end{array}$ \\
\hline TUCANO & TUCANO & ESPAÑOL & ESPAÑOL & ESPAÑOL & SIRIANO & ESPAÑOL & TUCANO & SIRIANO & $\begin{array}{c}\text { NO } \\
\text { TIENE } \\
\text { HIJOS }\end{array}$ & $\begin{array}{c}\text { NO } \\
\text { REGISTRA }\end{array}$ \\
\hline 1 & 11 & 1 & 1 & 1 & 1 & 1 & 1 & 1 & 1 & 4 \\
\hline
\end{tabular}




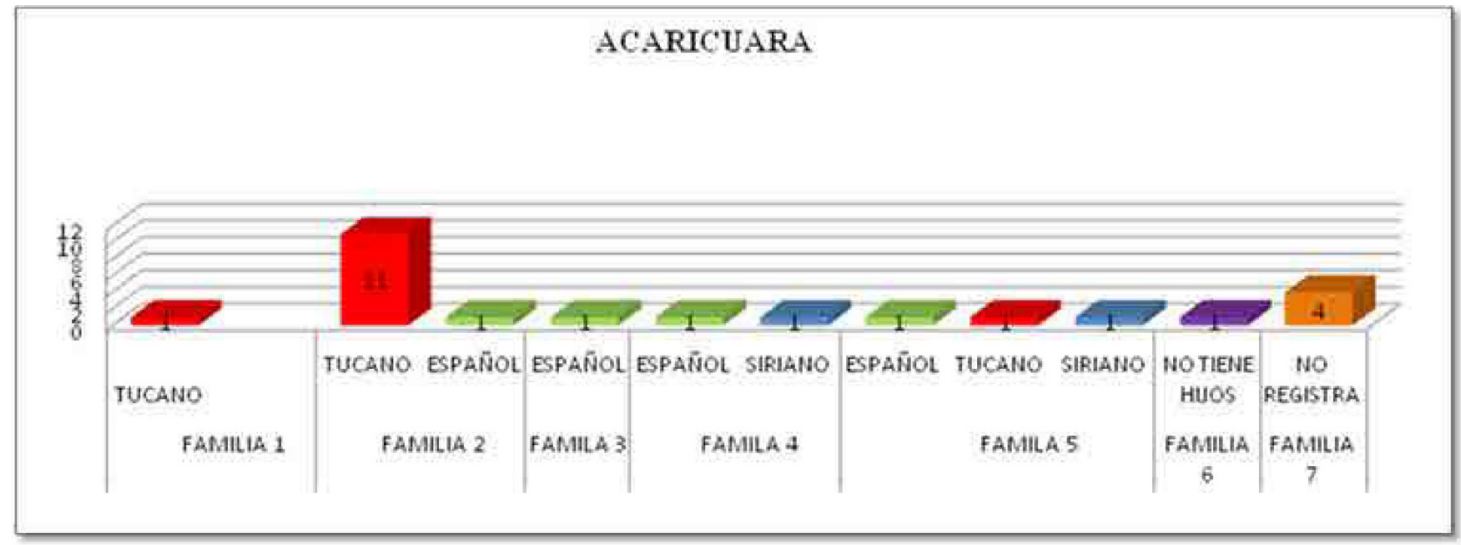

Figura 11. Lenguas aprendidas y habladas por los hijos, en Acaricuara.

En la Floresta sigue predominando la lengua tucana, seguida por el desano y el siriano.

\begin{tabular}{|c|c|c|c|c|c|}
\hline \multicolumn{5}{|c|}{ La Floresta } \\
\hline Familia 1 & \multicolumn{3}{|c|}{ Familia 2 } & \multicolumn{3}{c|}{ Familia 3 } \\
\hline Tucano & Desano & Español & Tucano & Tucano & Siriano \\
\hline 11 & 1 & 1 & 1 & 1 & 1 \\
\hline
\end{tabular}

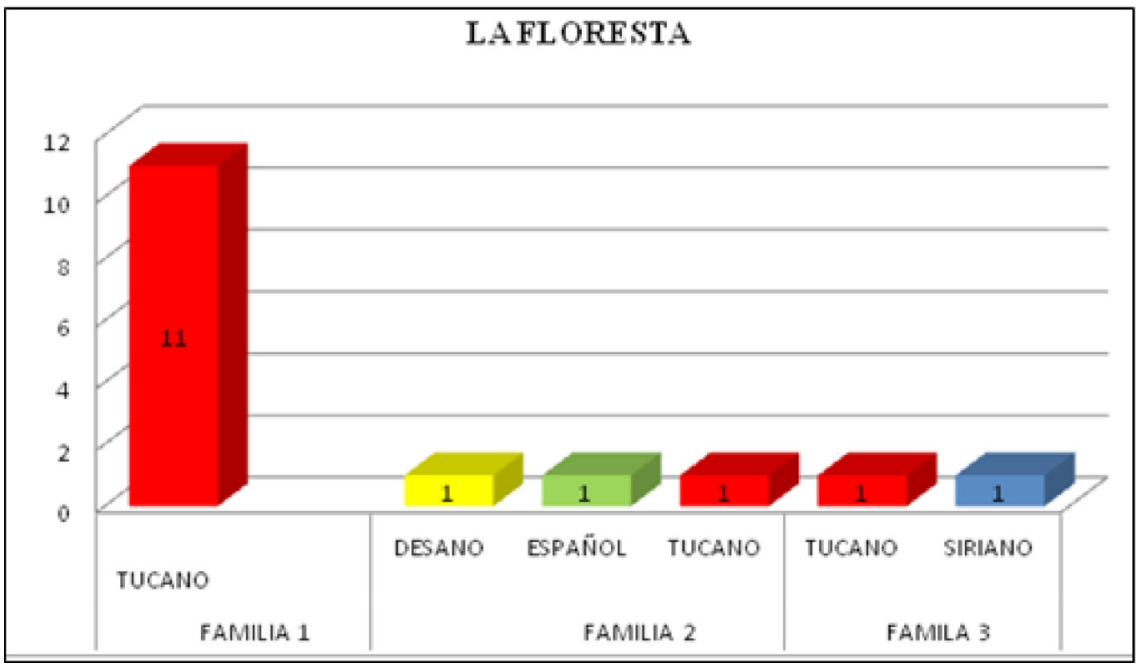

Figura 12. Lenguas aprendidas y habladas por los hijos, en La Floresta.

En Tamacuarí, los investigadores encontraron el uso del tucano seguido por el siriano. En términos generales, la cantidad de lenguas que hablan los hijos se restringen un poco en comparación con la cantidad de lenguas reportadas como habladas. Las que se mantienen son: tucano, siriano, desano y español, y de acuerdo con el actuar propio de la 
cultura, al continuar respetando la regla de la exogamia y el intercambio y aprendizaje de otras lenguas debe continuar.

\begin{tabular}{|l|l|}
\hline \multicolumn{2}{|c|}{ TAMACUARí } \\
\hline Familia 1 & Familia 2 \\
\hline Tucano & Siriano \\
\hline 10 & 1 \\
\hline & \\
\hline
\end{tabular}

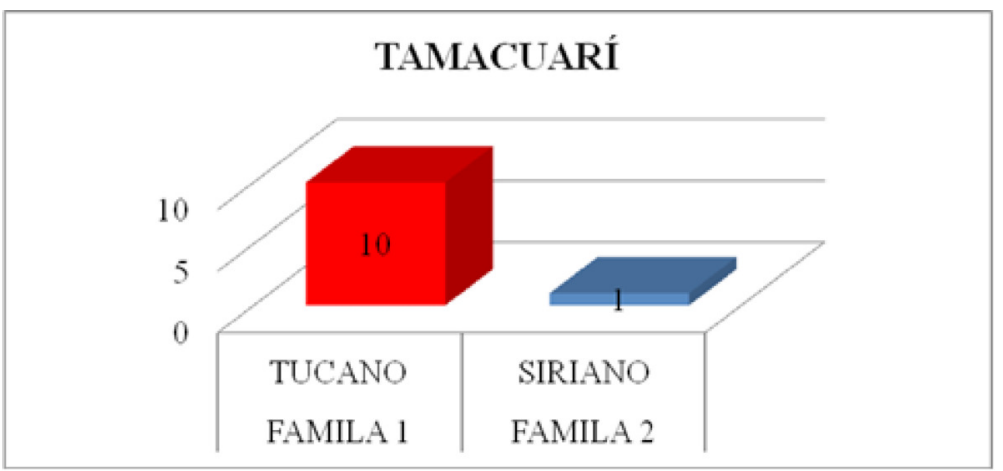

Figura 13. Lenguas aprendidas y habladas por los hijos, en Tamacuarí.

En la subcategoría lengua en la que estudian los hijos, se buscaba establecer si uno de los elementos básicos del proyecto etnoeducativo, como es el uso de la lengua materna, se empleaba en la escuela. Las cifras fueron los siguientes: en la Floresta, un $84 \%$ de los hijos reciben educación en español, en Acaricuara, un 75 \%, y en Tamacuari, un $64 \%$, el porcentaje restante corresponde a no estudian o no respondieron. Los resultados difieren con lo establecido en la Ley General de Educación (Congreso de la República de Colombia, 1994) en su artículo 5 que, en relación con la lengua, señala: "En sus respectivos territorios, la enseñanza de los grupos étnicos con tradición lingüística propia será bilingüe, tomando como fundamento escolar la lengua materna del respectivo grupo".

\begin{tabular}{|l|c|}
\hline \multicolumn{2}{|c|}{ ACUMULAD0 } \\
\hline Español & 34 \\
\hline No estudian & 7 \\
\hline No registra & 3 \\
\hline
\end{tabular}

\begin{tabular}{|l|c|}
\hline \multicolumn{2}{|c|}{ ACUMULADO } \\
\hline Español & $77 \%$ \\
\hline No estudian & $16 \%$ \\
\hline No registra & $7 \%$ \\
\hline
\end{tabular}




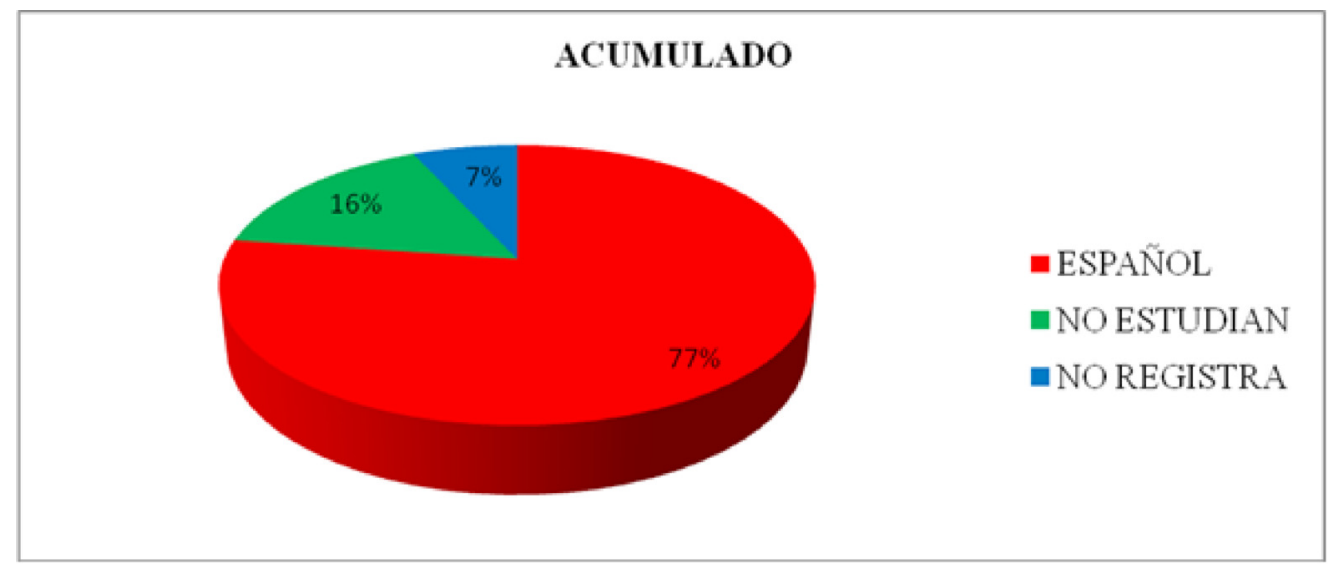

Figura 14. Lengua que se emplea en la escuela.

Los niños tucanos no están recibiendo una educación en lengua materna o una educación bilingüe, ya que la lengua empleada en la escuela es el español. La causa que origina el no recibir una educación en tucano, o una educación bilingüe, radica, según lo observado, en la falta de docentes tucanos o que hablen tucano (esta segunda opción no es nada sencilla, ya que hablar una lengua con la suficiencia requerida para enseñarla, implica recursos y un tiempo considerable).

Una vez más es posible determinar que no se cumple con lo estipulado en la Ley General de Educación, que en su artículo 59 determina:

El Gobierno Nacional a través del Ministerio de Educación Nacional y en concertación con los grupos étnicos prestará asesoría especializada en el desarrollo curricular, en la elaboración de textos y materiales educativos y en la ejecución de programas de investigación y capacitación etnolingüística. (Congreso de la República de Colombia, 1994, art. 59)

Además de la ausencia de profesores tucanos, se encuentra la falta de materiales didácticos en lengua materna; situación contraria a lo que establece la Ley General de Educación en su artículo 59:

El Gobierno Nacional a través del Ministerio de Educación Nacional y en concertación con los grupos étnicos prestará asesoría especializada en el desarrollo curricular, en la elaboración de textos y materiales educativos y en la ejecución de programas de investigación y capacitación etnolingüística. (Congreso de la República de Colombia, 1994, art. 59)

Si tenemos en cuenta que "Las posibilidades de preservar la gran riqueza lingüística y el multilingüismo en el Vaupés, dependerá en gran parte, de las políticas adoptadas en los programas educativos que se implementen entre los grupos" (Ardila (1993, p. 33), 
podemos afirmar que el panorama requiere de un cambio significativo en la forma en que se está enseñando en los colegios de las comunidades tucanas.

\section{Conclusiones}

A lo largo de la investigación y al analizar los resultados se evidenció que la etnia tucana continúa respetando la regla primigenia de la exogamia. No se reportaron casos de familias establecidas bajo relaciones endogámicas, y es posible afirmar que la exogamia mantiene vivo el multilingüismo en medio de la etnia tucana en el Vaupés.

Se pudo establecer que los miembros de la etnia tucana poseen la habilidad de comunicarse sin dificultad con miembros de las siguientes etnias: sirianos, desanos, tuyucas, barasanos, carapanas, yuruties, cubeos, guananos, piratapuyos, barás y curripacos, y aunque no lo registraron en las encuestas, se pudo observar al recorrer las comunidades tucanas ubicadas en el Brasil, que los tucanos colombianos que guiaron los viajes podían entender también el portugués.

Aunque pueden entender varias lenguas, para comunicarse entre ellos prefieren el tucano, seguido por el español, la lengua oficial, y el siriano, por ser la lengua que corresponde a la etnia más cercana.

En relación con los espacios de aprendizaje de las lenguas habladas por los tucanos (los mayores y los niños), el más recurrente es la casa, siendo tarea vigente y primordial de cada padre y madre enseñar la lengua propia a sus hijos; son conscientes de que al transmitir la lengua transmiten también de manera clara y directa los valores propios de su cultura.

Al consultar por los espacios y momentos de uso de las diferentes lenguas, los tucanos prefieren emplear la lengua materna en lugares como la casa, la chagra y las reuniones comunales, y prefieren usar el español en sitios como la iglesia, la escuela y Mitú.

Un espacio crítico en donde se evidenció la ausencia del uso de la lengua materna es la escuela. Las razones por las cuales no se está enseñando en tucano son: la ausencia de profesores tucanos o que hablen la lengua, la falta de material didáctico para la enseñanza en lengua materna y de un plan de estudios que responda a las necesidades de la comunidad educativa de la región y, por consiguiente, dé cumplimiento real a lo establecido en la Ley 115 de 1994 y el Decreto 804 de 1995 (normas que establecen los lineamientos de la etnoeducación).

Al dialogar con las personas de la etnia tucana se evidenció el respeto y el aprecio por su lengua, no sienten vergüenza por emplearla frente a extraños y consideran intere- 
sante que otros puedan aprender algo de ella. Aunque aprecian y usan su lengua y la de las comunidades vecinas, los tucanos son conscientes, y así lo manifestaron, de la necesidad que tienen sus hijos de un uso adecuado del español, especialmente para desenvolverse en ámbitos académicos y sociales fuera de sus comunidades.

Algunos líderes expresaron su preocupación por la pervivencia de las lenguas vernáculas en la región, y por ello consideran oportuno y necesario una educación realmente bilingüe, que les permita a sus hijos conservar la riqueza de su lengua y su cultura y, a la vez, contar con las herramientas necesarias para desarrollarse con eficiencia en medio de una sociedad mayoritaria en donde la lengua que predomina es el español. En otras palabras, poder vivir de manera equilibrada entre dos mundos.

\section{Recomendaciones}

Mantener el contacto con los líderes de la etnia tucana y establecer espacios que les permitan, con el apoyo necesario, elaborar materiales didácticos apropiados para la enseñanza en lengua materna.

Participar en espacios de reflexión que posibiliten el intercambio de ideas y la presentación de propuestas a las entidades territoriales encargadas de las políticas educativas en la región.

A medida que avance el conocimiento de lengua tucana, hay que profundizar en el estudio netamente lingüístico de sus características, ya que existen rasgos únicos y muy llamativos, como su sistema de clasificadores.

\section{Referencias}

Anokhina, 0. (2013). Estudios sobre multilingüismo y creación: eje prioritario de la política europea. París: Observatorio Europeo del Plurilingüismo.

Appel, R. \& Muysken, P. (1996). Bilingüismo y contacto de lenguas. Barcelona: Ariel.

Ardila, 0. (1993). Las lenguas tucano-orientales: estado actual y perspectivas de investigación. En Estado actual de la clasificación de las lenguas indígenas de Colombia. Bogotá: Instituto Caro y Cuervo.

Congreso de la República de Colombia (1994). Ley 115 de 8 de febrero de 1994, Ley General de Educación. Bogotá: Imprenta Nacional.

Constitución Política de la República de Colombia (1991). Anales del Congreso, 33(170), 15-37. 
Dwight, W. (1881), Mixture in Language. Philadelphia: Transactions, of the American Philosophical Association.

Fishman, J. (1995). Sociología del lenguaje. Madrid: Ediciones Cátedra.

Gaskell, G. (2002). Pesquisa qualitativa com texto, imagem e som. Río de Janeiro: Petropolis Vozes.

Grey, G. (1841). Journals of Two Expeditions of Discovery in North-West and Western Australia. Miami: Library of Alexandria.

Jackson, J. (1983). Identidad lingüística de los indios colombianos del Vaupés. Revista Lenguaje y Sociedad, 379-398.

Landaburu, J. (2004). La situación de las lenguas indígenas de Colombia: prolegómenos para una política lingüística viable. Amérique Latine Histoire et Mémoire. Les Cabiers ALHIM [En línea]. Recuperado de http://alhim.revues.org/125

Lévi-Strauss, C. (1985). Las estructuras elementales del parentesco. Barcelona: Planeta-Agostini.

López, L. (2000). El multilingüismo indo latinoamericano y la educación de la población indígena. Cochabamba: Documento de trabajo.

Ramírez, H. (2009). Interferencia y contacto de lenguas. Bogotá: Instituto Caro y Cuervo.

Sala, M. (1988). El problema de las lenguas en contacto. México: Universidad Nacional Autónoma de México.

Siguan, M. (2001). Bilingüismo y lenguas en contacto. Madrid: Alianza.

Silva-Corvalán, C. (1989). Sociolinguística teoría y análisis. Madrid: Alhambra.

Schuchardt, H. (1881). Die Cantes Flamencos. España: Fundación Machado.

Weinreich, U. (1974). Lenguas en contacto. Caracas: Universidad Central de Venezuela.

West, B. \& Welch, B. (2004). Gramática pedagógica del tucano. Bogotá: Fundación para el Desarrollo de los Pueblos Marginados. 